Research Paper

\title{
Multiple guided technologies based on radial probe endobronchial ultrasound for the diagnosis of solitary peripheral pulmonary lesions: a single-center study
}

\author{
Huang Haidong ${ }^{1 *}$, Ning Yunye ${ }^{1^{*}}$, Zhang Wei ${ }^{1^{*}}$, Paul Zarogoulidis ${ }^{2 凶}$, Wolfgang Hohenforst-Schmidt ${ }^{3}$, \\ Yan-Gao Man, Yang Yuguang5, Dong Yuchao, Bai Chong ${ }^{1 凶}$ \\ 1. Department of Respiratory and Critical Care Medicine, Changhai Hospital, The Second Military Medical University, Shanghai 200433, China. \\ 2. Pulmonary Department-Oncology Unit, "Theageneio" Anticancer Hospital, Thessaloniki, Greece. \\ 3. Sana Clinic Group Franken, Department of Cardiology / Pulmonology / Intensive Care / Nephrology, "Hof" Clinics, University of Erlangen, \\ Hof, Germany. \\ 4. International Union for Difficult-to-treat-Diseases (IUDD), MD, USA \\ 5. Department of anesthesiologist, Changhai Hospital, The Second Military Medical University, Shanghai 200433, China. \\ *Huang Haidong, Ning Yunye, Zhang Wei contributed equally to this work.
}

$\triangle$ Corresponding author: 1) Paul Zarogoulidis, M.D, Ph. D, Pulmonary Department-Oncology Unit, "Theageneio" Anticancer Hospital, Thessaloniki, Greece, Mobile: 00306977271974, E-mail: pzarog@hotmail.com. 2) Bai Chong, MD, Ph. D, Department of Respiratory and Critical Care Medicine, Changhai Hospital, The Second Military Medical University, Shanghai, China, E-mail: bc7878@sohu.com

(c) Ivyspring International Publisher. This is an open access article distributed under the terms of the Creative Commons Attribution (CC BY-NC) license (https://creativecommons.org/licenses/by-nc/4.0/). See http://ivyspring.com/terms for full terms and conditions.

Received: 2017.03.11; Accepted: 2017.08.31; Published: 2017.09.29

\begin{abstract}
Objective To evaluate the value of multiple guided technologies based on radial probe endobronchial ultrasound (R-EBUS) in the application of the diagnosis of solitary pulmonary peripheral lesions (PPLs).

Methods Analysis of diagnostic yield in 4 groups patients with different combined multiple guided technologies based on R-EBUS, 94 patients with 94 solitary PPLs from Mar, 2013 to Nov, 2014 in Changhai Hospital.

Results The overall diagnostic yield was $75 \%$ (70/94), the diagnostic yield of Group A (R-EBUS) was $62 \%(16 / 26)$, Group B (R-EBUS with guided sheath, EBUS-GS) was $76 \%$ (34/45), Group C (EBUS-GS with fluoroscopy) was $82 \%(9 / 11)$, Group D (virtual bronchoscopic navigation guided EBUS-GS with fluoroscopy) was $92 \%$ (11/12). The overall histopathological diagnostic yield was $56 \%$ (53/94. Better histopathological diagnostic yield was associated with application of multiple guided technologies based on EBUS-GS, lesions located in non-lower lobes, lesion's diameter > $2 \mathrm{~cm}$, radial probe within the lesions and lidocaine nebulization combined with intravenous anesthesia. There were no severe complications in all the 94 cases. A ultrasonic radial probe was broken when exploring a lesion located in the apical-posterior segment of left upper lobe.

Conclusion Multiple guided technologies based on R-EBUS were safe and effective in the diagnosis of solitary PPLs. In practice, the diagnosis yield improved with the application of forcep biopsies combined with bronchial brushing and washing.
\end{abstract}

Key words: bronchoscope, endobronchial ultrasound, radial probe, virtual bronchial navigation (VBN), EBUS-GS, fluoroscopy, peripheral pulmonary lesion, biopsy, diagnosis

\section{Introduction}

In recent years, radial probe endobronchial ultrasound (R-EBUS) has been increasingly used in transbronchial biopsy (TBBx) for peripheral pulmonary lesions (PPLs) with the development of endosonography technology. R-EBUS is now recommended by the ACCP lung cancer guidelines due to its high sensitivity without compromising safety.[1] Although R-EBUS is an effective method for 
the localization of PPLs, guiding the probe to the smaller lesion could be challenging. $[2,3]$ Other multiple guided technologies such as fluoroscopy (FLUO), R-EBUS with guide sheath (EBUS-GS), virtual bronchoscopy navigation (VBN), and Electromagnetic Navigation (EMN), could enhance the diagnostic yield of R-EBUS for PPLs.[2-6] With the continuous improvement of the positive diagnostic yield, natural orifice translumenal endoscopic surgery (NOTES) has gradually become the focus of clinical research. However, there are still rare clinical reports in China on higher positive diagnostic yield and lower level controlled risk of PPLs diagnosed by the combination of the guided technologies mentioned above. Therefore, we conducted a single-center retrospective study on R-EBUS-based guided technologies for solitary PPLs in Changhai Hospital. The present study was designed to assess the diagnostic yield, efficiency and safety of different combinations of guided technologies, which is beneficial for the clinical experience summary. Moreover; we added information regarding the methods and application of anesthesia, since the method of anesthesia plays a role in the efficiency of the diagnostic procedure.

\section{Patients and Methods}

\section{Patients}

Ninety four patients with solitary PPLs were diagnosed by radial endobronchial ultrasound (R-EBUS) combined with multiple guided technologies from March, 2013 to November, 2014. In our study all cases were retrospectively analyzed. The study population consisted of 34 outpatients and 60 hospitalized patients. In specific 64 patients were males and 30 females aged from 29 to 86 years with a mean age of $60.0 \pm 11$ years old. There were 94 pulmonary solitary lesions in the 94 patients enrolled. The principal inclusion criteria were as follows: (1) a diagnosis of solitary PPLs by chest computed tomography (CT); (2) presence of bronchioles around the lesion; and (3) no detection of endobronchial lesions by flexible bronchoscope. Exclusion criteria included: (1) severe cardiac insufficiency; (2) severe coagulation disorders; and (3) inability to tolerate the procedure of flexible bronchoscopy. Written informed consents were obtained from all the subjects and the study was approved by the Ethics Committee of Changhai Hospital, Second Military Medical University, Shanghai, China.

\section{Methods}

The patients should complete 6-8 hours of fasting, routine blood test, thin-slice CT scans of lungs, and electrocardiogram before the examination.
The procedures of bronchoscopy and endobronchial ultrasound were performed by 3 experienced associate interventional pulmonologists. Before the procedure, the 3 interventional pulmonologists studied pulmonary lung CT image data and divided the patients into 4 groups depending on lesion size, distribution of peripheral bronchus and complexity of path to lesion. The 4 groups were as follows: Group A (R-EBUS), Group B (EBUS-GS group), Group C (EBUS-GS+FLUO), Group D (VBN+EBUS-GS+ FLUO). When operating, the manipulator placed the insertion end of the bronchoscope at the nearest segmental bronchus from the lesion in the guidance of the CT image, and then inserted the R-EBUS probe through the working channel of scope. After probe entering the bronchus, opened ultrasound machine for scanning the segmental bronchus in target area. When the lesion was detected by radial ultrasound probe, its size was also measured. The procedures were performed as described below. As for Group A, the insertion end of the bronchus was fixed after exiting the radial probe, the biopsy forceps were used to get 4-6 tissue blocks for pathological examination through the working channel according to the depth of radial probe reached and the lesion size. Then, 2-3 times by bronchial brushing were performed for exfoliated cells, acid-fast bacilli. Finally, 40-60ml saline washing and lavage fluid was selected for the test of bacteria, fungi, acid-fast bacilli and exfoliated cells. As for Group B, after detecting the lesion by EBUS-GS, adjusted the guide sheath and put the distal end of sheath at the proximal site of the target lesion. Exited the radial probe. Retained and fixed guide sheath. The labeled biopsy forceps were inserted through lumen of guide sheath to achieve 4-6 tissue blocks for pathological examination. Then, labeled brush was inserted through the sheath for 2-3 times brushing for the test of exfoliated cells, acid-fast bacilli. Injected $40-60 \mathrm{ml}$ saline through sheath. Finally, pulled out the sheath and recovered lavage fluid for the test of bacteria, fungi, acid-fast bacilli and exfoliated cells. The procedures of Group C and Group B were essentially identical. However, unlike Group B, the biopsy and brushing examination of Group C were performed under X-ray fluoroscopy. As for Group D, the patient was checked by the thin-slice pulmonary CT scans (image interval $0.5-1 \mathrm{~mm}$ ) before procedure. Copied the data to the specified DICOM format disc and imported the data into VBN system for generation of electronic path. the manipulator placed the insertion end of the bronchoscopy at the nearest segmental bronchus from the lesion in the guidance of VBN path. The subsequent procedures were the same as that of Group C. The patients should be observed for 2 hours 
in the post-procedure room after procedures. Conducted re-examination of Chest X-ray to exclude pneumothorax. Then, recorded the complications such as hemoptysis and pneumothorax. Finally, analyzed the diagnostic yield of the patients after the return of the examination results. For patients with unknown diagnosis, confirmed the final diagnosis by percutaneous PPL biopsy, surgical pathology and follow-up after treatment.

The "painless" anesthesia, intravenous anesthesia combined with oropharyngeal lidocaine nebulization, was preferred for the patients after evaluation of the anesthesiologist. Patient was given oropharyngeal anesthesia by oral inhalation of $2 \mathrm{ml}$ of $2 \%$ lidocaine injection. Then, the patient was administered inducing dose of dexmedetomidine (1 $\mu \mathrm{g} / \mathrm{kg}$ ) via micro pump of superficial vein in 10-15 minutes. After that, the dose of $0.5-0.7 \mu \mathrm{g} \cdot \mathrm{kg}^{-1} \mathrm{~h}^{-1}$ was given for maintenance. After falling asleep, the patients were administrated midazolam (0.03-0.05 mg.kg-1) and fentanyl (25-50 $\mu \mathrm{g})$ through slow intravenous push. If necessary, the dosage of midazolam and fentanyl can be increased as appropriate. Inserted nasopharyngeal tube to keep the upper respiratory tract unobstructed. For the patients with contraindications, such as uncontrolled hypertension and arrhythmia, local anesthesia of oropharyngeal inhaled aerosolized lidocaine should be only applied alternatively. The method of anesthesia plays a crucial role for both the safety of the patient and efficiency of the diagnostic examination. In specific; keeping the airway open with adequate respiration is very important for the patient, but more importantly you need to control the respiratory rate. In the case of small pulmonary nodules the respiratory rate controls in a way the position of the solitary nodule at the crucial moment when performing the biopsy. The anesthesia that you administer to the patient or the method whether it is local or general is also associated with the health status of the patient that you are going to treat and the site of the nodule.

All procedures were performed using electronic flexible bronchoscope (1T260, Olympus Medical Systems Co. Ltd, Tokyo, Japan) and/or ultrasonic host (EU-ME1) universal ultrasonic endoscope image processing device, Olympus Medical Systems Co. Ltd., Tokyo, Japan). Other equipment and accessories included endobronchial ultrasound radial probe with a diameter of $1.7 \mathrm{~mm}$ (UM-S20-20R, Olympus Medical Systems Co. Ltd., Tokyo, Japan), disposable guide sheath with a diameter of $2.5 \mathrm{~mm}$ for radial probe (Olympus thick guide sheath, Olympus Medical Systems Co. Ltd., Tokyo, Japan), C-arm X-ray machine (Siemens ARCAPIS Varic, Siemens
Aktiengesellschaft, Berlin and Munich, Germany), Virtual bronchoscopy system (DirectPath 1.0, Cybernet systems Co. Ltd., Tokyo, Japan), disposable biopsy forceps (Nanjing Micro-tech Medical Science and Technology Co., Ltd., Nanjing, China) and cell brush (Nanjing Micro-tech Medical Science and Technology Co., Ltd., Nanjing, China).

\section{Statistical analysis}

Quantitative value was expressed as mean \pm standard error of mean $(\bar{x} \pm s)$, while enumeration value was indicated by percentage (\%). The analyses were conducted using SPSS version 21.0 (IBM SPSS Statistics, IBM Corporation, Armonk, New York, USA). Statistical analyses were performed by chi-square test. In all cases, $P$-values $<0.05$ were considered statistically significant.

\section{Results}

\section{Baseline characteristics and pathological results}

The 94 patients consisted of 34 outpatients and 60 hospitalized patients, which included 64 males and 30 females aged from 29 to 86 years with a mean age of $60 \pm 11$ years old. According to the final pathological results and clinical diagnosis, 70 (75\%) cases were diagnosed by combined multiple guided technologies successfully. In total, there were 70 patients with malignant disease, including 47 cases of adenocarcinoma, 7 cases of squamous cell carcinoma, 4 cases of small cell lung cancer, 4 cases of non-small cell lung cancer, 2 cases of metastatic carcinoma, 4 cases of atypical hyperplasia, 1 case of leiomyosarcoma, and 1 case of lymphoma. In addition, there were 18 patients with benign disease, including 5 cases of organizing pneumonia, 4 cases of pneumonia, 1 case of interstitial pneumonia, 1 case of organizing pneumonia complicated with bronchiolitis, 3 cases of tuberculosis, 3 cases of pulmonary aspergillosis, and 1 case of hamartoma. There were 6 outpatients with inconclusive diagnosis lost to follow-up.

Table 1. Baseline characteristics of the study patients

\begin{tabular}{ll}
\hline Characteristics & $\mathbf{n}$ \\
\hline Outpatient & 34 \\
Hospitalized patient & 60 \\
Mean age, range $(\overline{\mathbf{x}} \pm \mathbf{s})$ & $29-86(60.0 \pm 11)$ \\
Sex & \\
Male & 64 \\
Female & 30 \\
Pathological results & \\
Malignant & 70 \\
Benign & 18 \\
Inconclusive & 6 \\
\hline
\end{tabular}




\section{Size and distribution of PPLs}

Among the 94 patients, there were 94 PPLs with various sizes $(3-50 \mathrm{~mm})$. The lesions had a mean diameter of $24 \pm 13 \mathrm{~mm}$ under pulmonary CT scans. The distribution of lesions was as follows: 2 lesions in apicoposterior segment of left upper lobe, 7 lesions in the anterior segment of left upper lobe, 6 lesions in the lingular segment of the left upper lobe, 6 lesions in the superior segment of left lower lobe, 2 lesions in the medial anterior basal segment of left lower lobe, 4 lesions in the lateral posterior basal segment of left lower lobe, 6 lesions in the apical segment of right upper lobe, 10 lesions in the posterior segment of right upper lobe, 12 lesions in the anterior segment of right upper lobe, 8 lesions the lateral segment of in right middle lobe, 9 lesions the medial segment of in right middle lobe, 3 lesions in the superior segment of right lower lobe, 3 lesions in the medial basal segment of right lower lobe, 5 lesions in the anterior basal segment of right lower lobe, 8 lesions in the lateral basal segment of right lower lobe, 3 lesions in the posterior basal segment of right lower lobe.

Table 2. Distribution of solitary lesions in the peripheral lung fields

\begin{tabular}{ll}
\hline Segment & Number of solitary lesions \\
\hline left upper lobe & 2 \\
apicoposterior segment & \\
anterior segment & 7 \\
lingular segment & 6 \\
left lower lobe & \\
dorsal segment & 6 \\
medial anterior basal segment & 2 \\
lateral posterior basal segment & 4 \\
right upper lobe & \\
apical segment & 6 \\
posterior segment & 10 \\
anterior segment & 12 \\
right middle lobe & \\
lateral segment & 8 \\
medial segment & 9 \\
right lower lobe & \\
superior segment & 3 \\
medial basal segment & 3 \\
anterior basal segment & 5 \\
lateral basal segment & 8 \\
posterior basal segment & 3 \\
\hline
\end{tabular}

\section{Diagnostic yield}

Among the 4 groups, the diagnostic yield of Group D was $92 \%(11 / 12)$, higher than the other 3 groups (Table 3). The diagnostic yield of histopathology was 56\% (53/94). The exfoliated cells, acid-fast bacilli, bacteria and fungi were obtained by combination of brushing and lavage. And the diagnostic yield combined with cytology, histology and etiology was $75 \%$ (70/94).
Table 3. Diagnostic yield of 4 groups

\begin{tabular}{llll}
\hline Group & $\begin{array}{l}\text { Number of patients } \\
\text { enrolled }\end{array}$ & $\begin{array}{l}\text { Number of patients with } \\
\text { definitive diagnosis }\end{array}$ & $\begin{array}{l}\text { Diagnostic } \\
\text { yield (\%) }\end{array}$ \\
\hline Group A & 26 & 16 & 62 \\
Group B & 45 & 34 & 76 \\
Group C & 11 & 9 & 82 \\
Group D & 12 & 11 & 92 \\
\hline
\end{tabular}

\section{Other factors affecting diagnostic yield}

The statistical analysis of chi-square test showed no significant differences between the diagnostic yield of Group A, B, C, and D. However, the statistical results can also be affected by the insufficient sample numbers. Moreover, the clinical data revealed that the percentage rate of diagnostic yield in Group D was highest $(92 \%, 11 / 12)$. As for the patients whose pathological samples cannot be obtained by single method, combination of multiple methods can be used. The combination of brushing and washing can also be the factors affecting the diagnostic yield of biopsy forceps. The diagnostic yield of histopathological biopsy was 56\% (53/94). Interestingly, the diagnostic yield increased to $75 \%$ (70/94) when combining cytology and etiological examination by brushing and/or washing. The difference was statistically significant $\left(\left(X^{2}=6.80, P=\right.\right.$ 0.009). Subgroup analysis showed that the factors affecting the diagnostic yield also included location, size, and ultrasonic manifestations of lesion, whether combining with combined multiple guided technologies with guide sheath and whether using combination of intravenous anesthesia and inhalation anesthesia. The histopathological diagnostic yield of the lesions located in the upper and middle lobe was $60 \%(36 / 60)$. And, the histopathological diagnostic yield of right middle lobe lesions was highest $(77 \%$, 13/17). By contrast, the histopathological diagnostic yield of the lower lobe lesions was 35\% (12/34). The difference was statistically significant $\left(\chi^{2}=5.30, P=\right.$ 0.21). Under the CT scans, the histopathological diagnostic yield of PPLs with diameter less than $2 \mathrm{~cm}$ was 56\% (15/27), while that of PPLs with diameter greater than $2 \mathrm{~cm}$ was $82 \%$ (55/67). The difference was statistically significant $\left(X^{2}=7.13, P=0.008\right)$. Under ultrasonoscopy, the histopathological diagnostic yield was $67 \%(42 / 63)$ when the miniprobe is located within the lesion. When the radial probe was located at the edge of lesion, the histopathological diagnostic yield was $38 \%(6 / 16)$. The difference was statistically significant $\left(X^{2}=4.55, P<0.05\right)$. The histopathological diagnostic yield of groups (Group B, C, and D) with sheath was $47 \%$. The group without sheath (Group A) was $23 \%(6 / 26)$. The difference was statistically significant $\left(\chi^{2}=4.49, P=0.034\right)$. In the patients using combination of intravenous anesthesia and inhalation 
of lidocaine, the histopathological diagnostic yield was $63 \%(41 / 65)$. When inhalation anesthesia was applied alone, the histopathological diagnostic yield was $38 \%(11 / 29)$. The difference was statistically significant $\left(X^{2}=5.13, P=0.024\right)$.

\section{Complications}

No pneumothorax occurred in all patients. In procedures, only 2 patients had hemoptysis estimated total amount of $20-30 \mathrm{ml}$ blood. After endobronchial washing with ice saline into the lesion, the bleeding stopped. When detecting with R-EBUS in one patient with lesion in left upper lobe apicoposterior segment, the tip of ultrasonic radial probe had been broken and destroyed.

\section{Discussion}

As early as in 1970s, several interventional pulmonologists from Europe and the United States have begun to carry out TBBx for PPLs under FLUO. It was not widely promoted in clinical practice due to its low diagnostic yield.[7-9] In recent years, varies of transbronchial guided technologies including R-EBUS are increasingly mature. However, the diagnostic yield with single technology is still low (40\%-80\%).[10-17] Therefore, the clinical application is limited. To enhance the diagnostic yield of PPLs, interventional pulmonologists turn to combining multiple technologies.[18-22] It was reported that the diagnostic yield can increase to $67 \%-81 \%$ when combined with GS-EBUS, FLUO, and VBN.[18, 19] The study of Tamiya et. al. [23] showed that the combination of EBUS-GS and VBN was useful for diagnosing small PPLs $(\leq 3 \mathrm{~cm})$ without compromising safety. The diagnostic yield was $83.7 \%$ and $68.0 \%$ for the malignant and benign lesions, respectively(23). However, a systematic review and meta-analysis including 1,033 cases showed that the diagnostic yield of combination of EBUS-GS, FLUO, and VBN was only 75\%.[5] This was not significantly different from the diagnostic yield of combination of VBN, EBUS-GS, and FLUO. Our results showed that the overall diagnostic yield of 94 patients was $75 \%$ (70/94). The diagnostic yield was high due to the combination of biopsy, washing and brushing. In comparison, the histopathological diagnostic yield was only $56 \%$ (53/94) when biopsy pathology was applied alone. The difference was statistically significant $(X 2=6.80, P=0.009)$. Cytology detection of exfoliated cells improved the diagnostic yield of tumors while etiology detection improved the diagnostic yield of infectious diseases such as tuberculosis and aspergillus.

In addition, subgroup analysis showed that the selection of appropriate patients was associated with higher histopathological diagnostic yield. The patients with higher histopathological diagnostic yield should have following characteristics: non-lower lobe lesion, lesion diameter $>2 \mathrm{~cm}$, ultrasonic radial probe located in the interior of the lesion. Moreover, Gex et. al. [5] reported that the higher diagnostic yield was associated with following factors: upper or middle lobe lesion, lesion diameter > $2 \mathrm{~cm}$, bronchial sign under CT scan. These results are basically consistent with our research. In our study, the percentage rate of diagnostic yield in Group D (EBUS-GS, FLUO and VBN) was the highest, reaching $92 \%$, which was basically close to the diagnostic yield of CT-guided percutaneous PPLs biopsy. Clinical practice has proved that VBN cannot only prejudge if reaching PPLs, but also reduce the operating time, the wastage of ultrasonic radial probe and the dose of X-ray exposure significantly.[23, 24] As for one patient in Group A, only one suspicious solitary shadow under ultrasound image was found in the posterior basal segment of the right lower lobe after manipulated with EBUS-GS alone for 45 minutes (Figure 1). However, after imported the thin-slice CT data into VBN system, we could confirm that the lesion was located in the distal subsegment of the right lower lobe with about $8.32 \mathrm{~cm}$ deeply from the distal end of bronchus intermedius according to the guide path automatically generated by VBN (Figure 2). The second procedure took only 15 minutes to obtain the pathological tissue of PPLs, which was diagnosed as squamous cell carcinoma. The reason is that the radial probe was advanced into the segment of the bronchus lateral the lesion due to the high lung volume in lower lobe as well as large number of branches in the first procedure. In the second procedure, the guide path automatically generated by VBN leaded to the right sub segmental bronchus. Then, the guide sheath was advanced into the center of the lesion.

According to the literature, the incidence of combined multiple guided technologies procedure complicated with severe complications such as pneumothorax and massive hemoptysis was very low, which was generally less than $4 \%$, which was considerably lower than that of CT-guided percutaneous PPLs biopsy.[5] There were no severe complications in the 94 patients of our study. Only in one patient, the tip of radial probe had been broken and destroyed. It is worth pointing out that the ultrasonic radial probe is expensive with precise design of the mechanical part of in the distal end. Thus, the radial probe can be easily broken when advancing into segmental bronchus with large angle, especially in the apical segment of upper lobe, which is suggested to reduce the application of radial probe 
in detecting segmental bronchus with large angle during the procedure.

In addition, the compliance of patients during procedure is one of the key factors affecting diagnostic yield, especially when the ultrasonic radial probe reached the lesion, a slight cough or even slightly larger amplitude of respiratory action would lead displacement of the target lesion. Intravenous anesthesia can be used to make the patient in a moderate to deep sleep state, improving the patient's comfort and tolerance. Subgroup analysis showed that the combination of intravenous anesthesia with lidocaine nebulization significantly enhanced the histopathological diagnostic yield compared with the simple lidocaine nebulization. The application of intravenous sedation in bronchoscopy procedure is common in European as well as in the United States. Combined with lidocaine nebulization, intravenous anesthesia with midazolam and fentanyl as the leading drugs is also known as the "painless" anesthesia. In recent years, the "painless" anesthesia has been used in the domestic large respiratory endoscopy centers. The "painless" anesthesia can significantly reduce the discomfort feelings of patients in procedure and reduce the patient's cough and restlessness, improving efficiency. Therefore, it is recommended as the routine anesthetic method in the procedure of combined multiple guided technologies.[25]
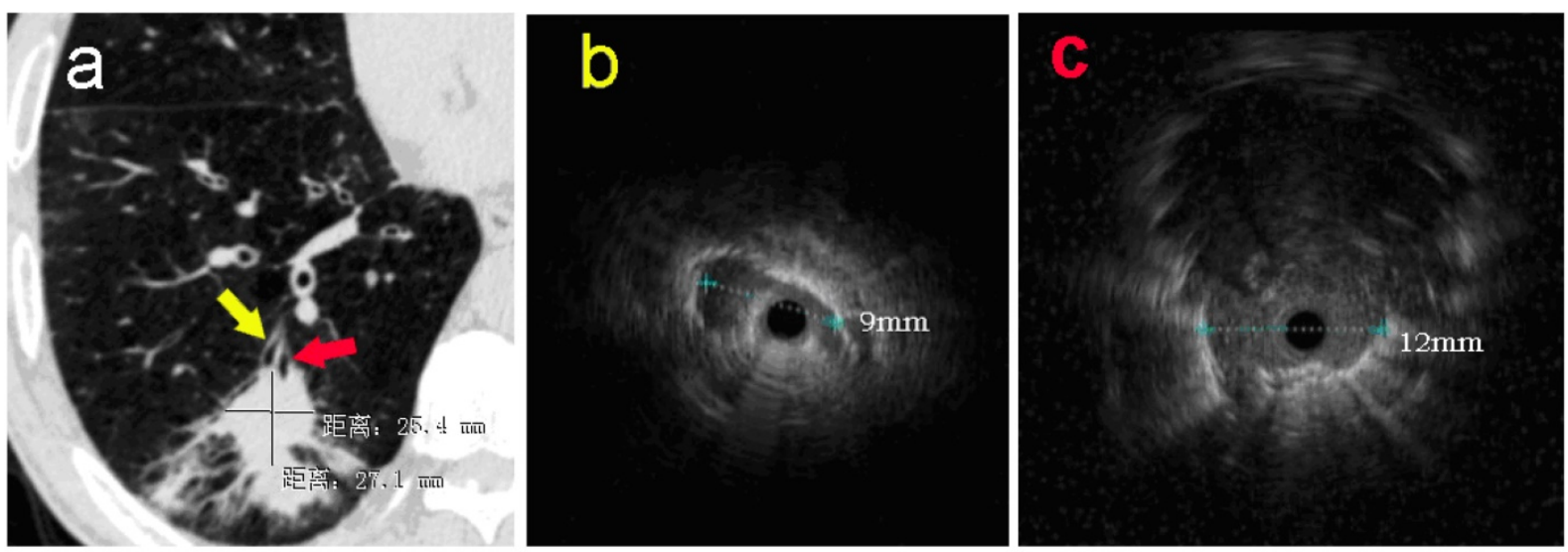

Figure 1 a: The pulmonary CT scan showed a nodule with estimated diameter about $27 \times 25 \mathrm{~mm}$ in basal segment of the right lower lobe. Without guidance of VBN, ultrasonic radial probe was advanced into the lateral branch of the lesion (yellow arrow). b: The ultrasonic image showed that only one strip suspicious solitary density shadow was found on the edge of the probe with the longest diameter of $9 \mathrm{~mm}$. In the second procedure, EBUS-GS was advanced into the center position of lesion (red arrow) in the posterior basal segment of right lower lung under the guidance of VBN 3D electronic path. c: Ultrasonic image showed the typical solidary circular density shadow with clear boundary edge with the longest diameter about $12 \mathrm{~mm}$. The radial probe was located within the lesion.

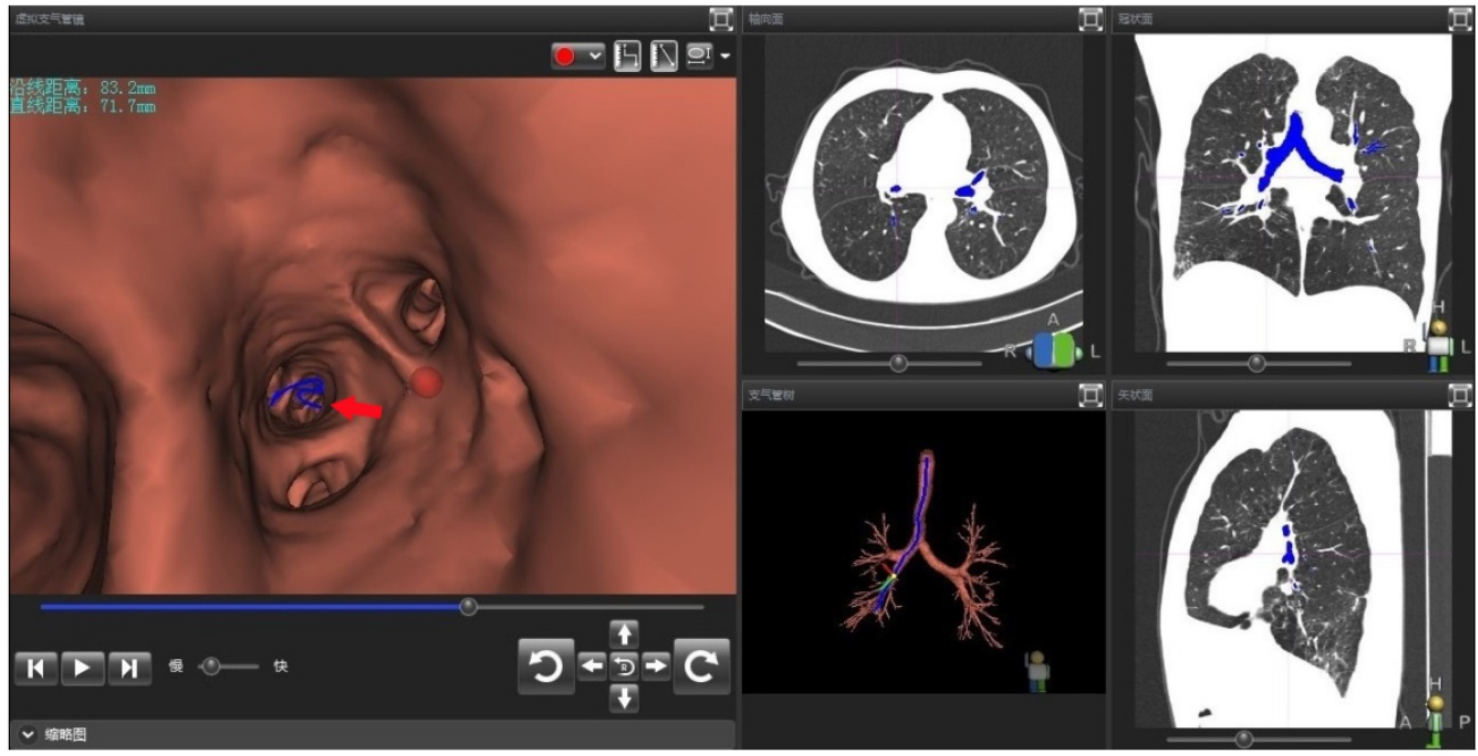

Figure 2 Operator interface of VBN DirectPath 1.0. The left figure referred to the virtual bronchoscopy image generated by VBN system automatically. In the virtual image, the position of red dot was the target lesion in the distal posterior basal segment of right lower lobe. The red arrow showed the electronic path leading to the lesion. The upper left text revealed the distance from lesion to distal end of bronchus intermedius was $83 \mathrm{~mm}$ in foot line and $72 \mathrm{~mm}$ in straight line. The right figure referred to the CT cross section, coronal plane, and sagittal plane and the dynamic path under the three-dimensional reconstruction of the tracheobronchial tree. 
In China, the clinical application of the combined multiple guided technologies is still in the initial stage.[26, 27] There are still many practical problems to be solved. For example, how to choose different combinations of guiding techniques for PPLs with different properties? How to set up the standard and optimize all kinds of transbronchial guided technology? How to reduce and avoid the unnecessary radiation exposure of patients and medical staff from X-ray? In our opinion, the problems listed above should be solved by conducting multi-center clinical trials, developing appropriate operating specifications and training program. The combination of intravenous anesthesia with oropharyngeal lidocaine nebulization significantly improved the efficiency of the procedure.

Currently, transbronchial ultrasound-based combined multiple guided technologies has developed into a safe and effective method for the diagnosis of PPLs. The high diagnostic yield can be obtained by combined multiple guided technologies with EBUS-GS as the leading technology. Moreover, the patients are well tolerated. Thus, it is recommended in general clinical practice. New technologies, such as EMN with expensive price and cumbersome procedure, are still gradually to mature. With the development of technology and clinical application, EMN will also further develop its potential to acquire small nodules or even micronodules in peripheral lung together with other combined multiple guided technologies. Major limitation in our was the small number of patients included, however; one should consider that these procedures even in centers with high experience are not performed routinely. Moreover; the number of patients that need these procedures even in a center of diagnostic excellence is low, since other non-interventional imaging procedures such as; PET-CT as an initial diagnostic tool is usually used. In the near future, it is believed that the R-EBUS based combined multiple guided technologies will potentially become the first choice for the diagnosis of PPLs in more and more centers. More importantly, it can provide technical support for the endobronchial interventional therapy for early stage peripheral lung cancer. Moreover; the new thin Convex Probe-EBUS from Oympus has just been tested in animals with a 25G needle and it has the ability to take samples from stations $1 \mathrm{~N}$ right and left. This new tool has different characteristics, firstly it does not need a balloon for the ultrasound, secondly; it has a smaller working channel and therefore biopsy needle and thirdly it is smaller in diameter and therefore it can go through smaller airways.[28] We trust that the application of R-EBUS based combined multiple guided technologies has a bright future in therapeutic bronchoscopic minimally invasive procedures.

\section{Acknowledgements}

This study was supported by Special Research Grants for China Non-Profit \& Public Service "Study on the diagnosis and treatment standard, criteria and evaluation of interventional pulmonary technology" (Grant number:201402024). Project of Wujieping Medical Foundation (Grant number: 320.6750.11112). Project of New Clinical Technology Development of Changhai Hospital (Grant number: NT201506).

\section{Competing Interests}

The authors have declared that no competing interest exists.

\section{References}

1. Rivera MP MA, Wahidi MM. Establishing the diagnosis of lung cancer: Diagnosis and management of lung cancer, 3rd ed: American College of Chest Physicians evidence-based clinical practice guidelines. . 3rd ed: CHEST; 2013.

2. Asano F. Recommendation of Combination of Radial Endobronchial Ultrasound With Virtual Bronchoscopic Navigation. Journal of bronchology \& interventional pulmonology. 2016; 23: 187-8. doi:10.1097/LBR.0000000000 000301.

3. Asano F. Advanced bronchoscopy for the diagnosis of peripheral pulmonary lesions. Respiratory investigation. 2016; 54: 224-9. doi:10.1016/j.resinv. 2015.11.008

4. Herth FJ, Ernst A, Becker HD. Endobronchial ultrasound-guided transbronchial lung biopsy in solitary pulmonary nodules and peripheral lesions. The European respiratory journal. 2002; 20: 972-4.

5. Gex G, Pralong JA, Combescure C, Seijo L, Rochat T, Soccal PM. Diagnostic yield and safety of electromagnetic navigation bronchoscopy for lung nodules: a systematic review and meta-analysis. Respiration; international review of thoracic diseases. 2014; 87: 165-76. doi:10.1159/000355710.

6. Matsumoto Y, Izumo T, Sasada S, Tsuchida T, Ohe Y. Diagnostic utility of endobronchial ultrasound with a guide sheath under the computed tomography workstation (ziostation) for small peripheral pulmonary lesions. The clinical respiratory journal. 2017; 11: 185-92. doi:10.1111/crj.12321.

7. Hanson RR, Zavala DC, Rhodes ML, Keim LW, Smith JD. Transbronchial biopsy via flexible fiberoptic bronchoscope; results in 164 patients. The American review of respiratory disease. 1976; 114: 67-72. doi:10.1164/arrd.1976.114.1.67.

8. Cortese DA, McDougall JC. Biopsy and brushing of peripheral lung cancer with fluoroscopic guidance. Chest. 1979; 75: 141-5.

9. Rivera MP MA, Wahidi MM. Initial diagnosis of lung cancer: ACCP evidence-based clinical practice guidelines. 2nd ed: CHEST; 2007.

10. Odronic SI, Gildea TR, Chute DJ. Electromagnetic navigation bronchoscopy-guided fine needle aspiration for the diagnosis of lung lesions. Diagnostic cytopathology. 2014; 42: 1045-50. doi:10.1002/dc.23164.

11. Kurimoto N, Miyazawa T, Okimasa S, Maeda A, Oiwa $H$, Miyazu $\mathrm{Y}$, et al. Endobronchial ultrasonography using a guide sheath increases the ability to diagnose peripheral pulmonary lesions endoscopically. Chest. 2004; 126: 959-65. doi:10.1378/chest.126.3.959.

12. Eberhardt R, Ernst A, Herth FJ. Ultrasound-guided transbronchial biopsy of solitary pulmonary nodules less than $20 \mathrm{~mm}$. The European respiratory journal. 2009; 34: 1284-7. doi:10.1183/09031936.00166708.

13. Kikuchi E, Yamazaki K, Sukoh N, Kikuchi J, Asahina H, Imura M, et al. Endobronchial ultrasonography with guide-sheath for peripheral pulmonary lesions. The European respiratory journal. 2004; 24: 533-7. doi:10.1183/09031936.04.00138603.

14. Yang MC, Liu WT, Wang CH, Lin HC, Chen HC, Chou CL, et al. Diagnostic value of endobronchial ultrasound-guided transbronchial lung biopsy in peripheral lung cancers. Journal of the Formosan Medical Association = Taiwan yi zhi. 2004; 103: 124-9.

15. Asahina H, Yamazaki K, Onodera Y, Kikuchi E, Shinagawa N, Asano F, et al. Transbronchial biopsy using endobronchial ultrasonography with a guide sheath and virtual bronchoscopic navigation. Chest. 2005; 128: 1761-5. doi:10.1378/chest.128.3.1761.

16. Herth FJ, Eberhardt R, Becker HD, Ernst A. Endobronchial ultrasound-guided transbronchial lung biopsy in fluoroscopically invisible solitary pulmonary nodules: a prospective trial. Chest. 2006; 129: 147-50. doi:10.1378/chest 129.1.147.

17. Yoshikawa M, Sukoh N, Yamazaki K, Kanazawa K, Fukumoto S, Harada M, et al. Diagnostic value of endobronchial ultrasonography with a guide sheath for 
peripheral pulmonary lesions without X-ray fluoroscopy. Chest. 2007; 131: 1788-93. doi:10.1378/chest.06-2506.

18. Ishida T, Asano F, Yamazaki K, Shinagawa N, Oizumi S, Moriya H, et al. Virtual bronchoscopic navigation combined with endobronchial ultrasound to diagnose small peripheral pulmonary lesions: a randomised trial. Thorax. 2011; 66: 1072-7. doi:10.1136/thx.2010.145490.

19. Asano F, Shinagawa N, Ishida T, Shindoh J, Anzai M, Tsuzuku A, et al. Virtual bronchoscopic navigation combined with ultrathin bronchoscopy. A randomized clinical trial. American journal of respiratory and critical care medicine. 2013; 188: 327-33. doi:10.1164/rccm.201211-2104OC

20. Chee A, Stather DR, Maceachern P, Martel S, Delage A, Simon M, et al. Diagnostic utility of peripheral endobronchial ultrasound with electromagnetic navigation bronchoscopy in peripheral lung nodules. Respirology. 2013; 18: 784-9. doi:10.1111/resp.12085.

21. Eberhardt R, Anantham D, Ernst A, Feller-Kopman D, Herth F. Multimodality bronchoscopic diagnosis of peripheral lung lesions: a randomized controlled trial. American journal of respiratory and critical care medicine. 2007; 176: 36-41. doi:10.1164/rccm.200612-1866OC.

22. Xu CH, Yu LK, Cao L, Yang R, Yan J, Liu ZC, et al. Value of radial probe endobronchial ultrasound-guided localization of solitary pulmonary nodules with the combination of ultrathin bronchoscopy and methylene blue prior to video-assisted thoracoscopic surgery. Molecular and clinical oncology. 2016; 5: 279-82. doi:10.3892/mco.2016.913.

23. Tamiya M, Okamoto N, Sasada S, Shiroyama T, Morishita N, Suzuki H, et al. Diagnostic yield of combined bronchoscopy and endobronchial ultrasonography, under LungPoint guidance for small peripheral pulmonary lesions. Respirology. 2013; 18: 834-9. doi:10.1111/resp.12095.

24. Sano A, Tsuchiya T. Virtual Bronchoscopy Using OsiriX. Journal of bronchology \& interventional pulmonology. 2014; 21: 113-6. doi:10.1097/ LBR.0000000000000051.

25. Jose RJ, Shaefi S, Navani N. Sedation for flexible bronchoscopy: current and emerging evidence. European respiratory review : an official journal of the European Respiratory Society. 2013; 22: 106-16. doi:10.1183/09059180 .00006412 .

26. Huang $Y$, Ren $\mathrm{HY}, \mathrm{He} B F, \mathrm{Li} X Y$, Chen $Z X$. [Non-real-time endobronchial bronchoscopy ultrasound assisted transbronchial lung biopsy in diagnosing peripheral pulmonary lesions]. Zhonghua jie he he hu xi za zhi $=$ Zhonghua jiehe he huxi zazhi $=$ Chinese journal of tuberculosis and respiratory diseases. 2013; 36: 12-6

27. Li M, Peng A, Zhang G, Song X, Li J, Tan M, et al. [Endobronchial ultrasound transbronchial lung biopsy with guide-sheath for the diagnosis of peripheral pulmonary lesions]. Zhonghua jie he he hu xi za zhi = Zhonghua jiehe he huxi zazhi $=$ Chinese journal of tuberculosis and respiratory diseases. 2014; 37: 36-40.

28. Wada $\mathrm{H}$, Hirohashi $\mathrm{K}$, Nakajima $\mathrm{T}$, Anayama $\mathrm{T}$, Kato $\mathrm{T}$, Grindlay $\mathrm{A}$, et al. Assessment of the new thin convex probe endobronchial ultrasound bronchoscope and the dedicated aspiration needle: a preliminary study in the porcine lung. Journal of bronchology \& interventional pulmonology. 2015; 22: 20-7. doi:10.1097/LBR.0000000000000123. 